\title{
The automatic pilot of the hand is unbalanced by visual neglect
}

\author{
Robert D. McIntosh ${ }^{\mathrm{a}, *}$, Stephanie Rossit ${ }^{\mathrm{b}}$, Paresh Malhotra ${ }^{\mathrm{c}}$, Stephen H. Butler ${ }^{\mathrm{d}}$ and Monika Harvey \\ ${ }^{\mathrm{a}}$ Human Cognitive Neuroscience, University of Edinburgh, Edinburgh, UK \\ ${ }^{\mathrm{b}}$ Department of Psychology, University of Western Ontario, Londen, Canada \\ ${ }^{\mathrm{c}}$ Division of Neurosciences and Mental Health, Imperial College London, London, UK \\ ${ }^{\mathrm{d}}$ School of Psychological Sciences and Health, University of Strathclyde, Glasgow, UK \\ e School of Psychology, University of Glasgow, Glasgow, UK
}

The automatic pilot of the hand refers to the capacity for fast in-flight correction of reaching. It is often studied using 'double-step' reaching tasks, in which the target is jumped to a new location once the reach is underway. Target-directed corrections occur even if people are not instructed to follow the target (GO task), but are asked instead to stop the reach if they see the target jump (STOP task), suggesting that such corrections are a default visuomotor behaviour [9]. Uninstructed corrections in the STOP task were absent in a patient with optic ataxia following bilateral parietooccipital lesions [9], implying that they depend upon the visuomotor dorsal stream.

Visual neglect after right hemisphere damage is most strongly associated with temporo-parietal lesions, inferior to the dorsal stream. It has thus been proposed that, in many patients with neglect, direct visuomotor behaviour should be free from the perceptual biases that characterise the syndrome [8]. Consistent with this, neglect does not seem to entail specific biases in reaching or grasping [1-3,5]. Moreover, patients may plan their reaches to avoid obstacles on the neglected side [6]; and a patient with visual extinction was shown to avoid obstacles, and to use online visual feedback from the hand, even when he could not report the relevant stimuli $[7,11]$. On the other hand, one study was

\footnotetext{
*Corresponding author: Robert D. McIntosh, Psychology, University of Edinburgh 7 George Square, Edinburgh, EH8 9JZ, United Kingdom. Tel.: +44 131650 3444; Fax: +44 131650 3461; E-mail: r.d.mcintosh@ed.ac.uk.
}

able to isolate a motor-related component to neglect. Specifically, Mattingley et al. [4] demonstrated a retarded initiation of leftward movements into left hemispace in patients with neglect following inferior parietal lobe lesions.

We studied online visuomotor reactions to target jumps during double-step reaching in seven right-braindamaged patients with neglect (RBDN; mean age 68.1 years, SD 9.5), eight right-brain-damaged controls without neglect (RDBC; mean age 62.5 years, SD 10.3), and eight healthy controls (HC; mean age 72.9 years, SD 4.0). Participants reached with the right index finger $40 \mathrm{~cm}$ forwards for a central target dot of $7 \mathrm{~mm}$ diameter, which remained static ( $70 \%$ of trials) or jumped at movement onset by $4 \mathrm{~cm}$ to the left $(15 \%$ of trials) or right ( $15 \%$ of trials). In separate blocks of 200 trials, the instruction was to follow the target (GO task) or to stop the movement (STOP task) if the target jumped, with block order counterbalanced across participants. Movements were recorded from the finger at $108 \mathrm{~Hz}$, using an electro-magnetic tracking system (MiniBIRD). For each participant, a spatial bandwidth for reaches to central targets was set at 2.81 SD either side of the average $2 \mathrm{D}$ hand path on static trials. For each frame of each jump trial, the reach was classed as corrected if its coordinates lay beyond the bandwidth of the static trials, in the direction of the target jump, being otherwise classed as uncorrected.

Patients with neglect reacted to rightward jumps as rapidly as controls, but were abnormally slow to react to leftward jumps; this was true even for RBDN 

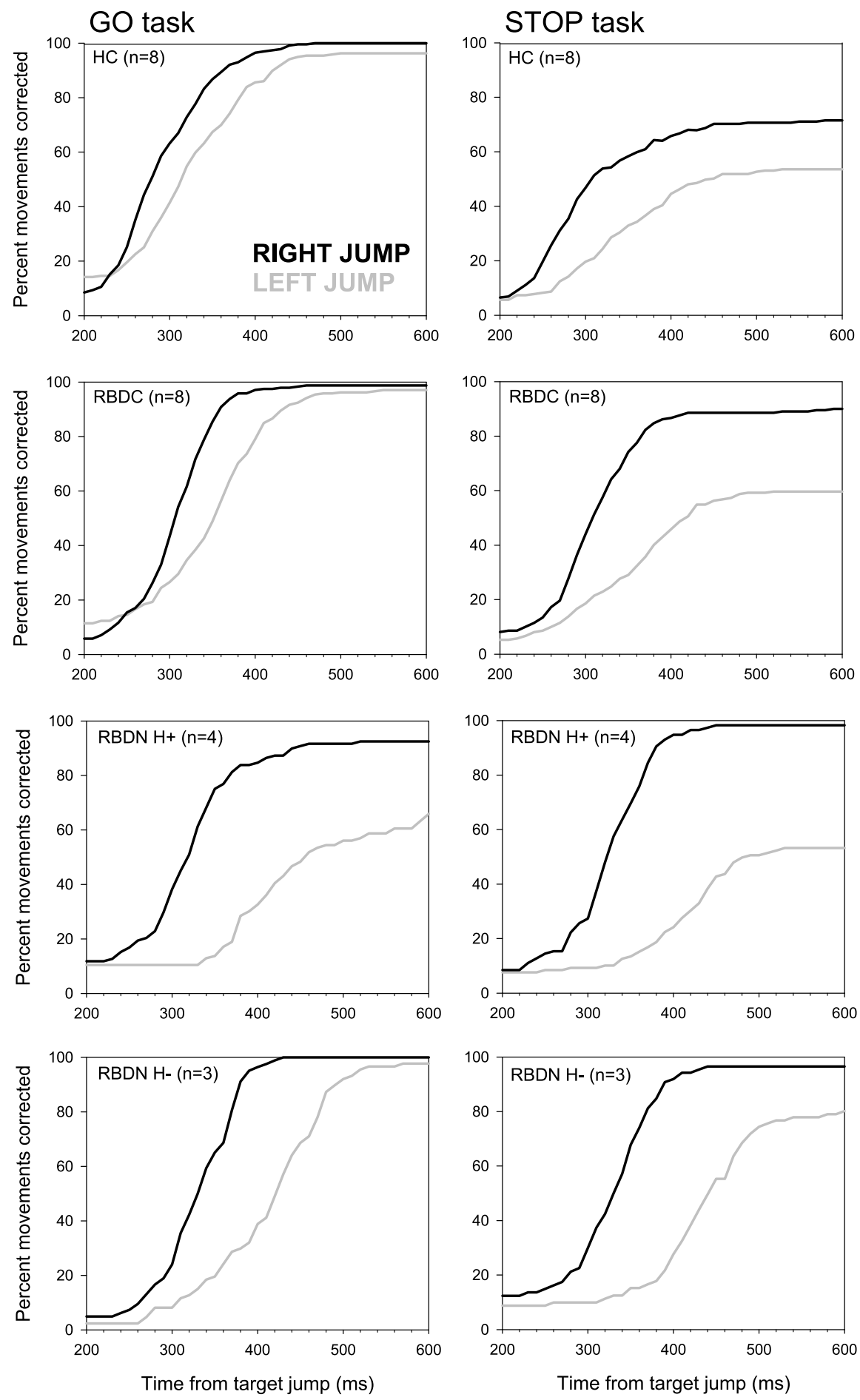

Fig. 1. Cumulative proportion of corrected responses on left jump (grey lines) and right jump (black lines) trials, by time from target jump, plotted up to $600 \mathrm{~ms}$ from target jump. Separate plots are shown for healthy controls (HC), right brain-damaged controls (RBDC), and right brain-damaged patients with neglect with hemianopia (RBDN H+) and without hemianopia (RBDN H-). The left column shows plots for the GO task, the right column for the STOP task. Both neglect sub-groups show abnormally late emergence of corrections to the left side. Nonetheless, corrections are made to the left even in the STOP task in which corrections are not instructed, implying integrity of the automatic pilot system, though with reduced responsiveness to the left side. 
patients without left visual field deficits (assessed by computerised perimetry), as shown in Fig. 1. Moreover, an analysis of movement time for successfully corrected movements revealed a group by side interaction $\left(\mathrm{F}_{2,19}=6.63, p<0.01\right)$, reflecting differentially longer duration movements to the left than to the right in the RBDN (727 vs. $617 \mathrm{~ms}$ ), as compared with the RBDC (639 vs. $581 \mathrm{~ms}$ ) and HC (593 vs. $561 \mathrm{~ms}$ ) groups. Nonetheless, neglect patients did make corrections to leftward target jumps, even in the STOP condition (Fig. 1). The occurrence of these uninstructed corrections suggest that the 'automatic pilot' system is functional in neglect; but it is unbalanced so that corrections to the left emerge abnormally slowly. This could conceivably be due to a retarded initiation of the corrective movement (a leftward movement into left hemispace [4]), but it seems more probable that the slowed correction to the left side is a consequence of inattention to the left, which reduces the salience of left-sided targets. We suggest that, although the automatic pilot system itself may be spared in neglect, its functioning is unbalanced by weakened attention to the left side. This implies shared attentional influences on vision-for-perception and vision-for-action, perhaps realised via top-down modulation of early visual areas.

An unexpected finding emerged from the analysis of the STOP responses themselves. The RBDN group had a dramatically impaired ability to stop their movements in response to a target jump to either side. A mixedmodel ANOVA comparing successful stop rates across groups for left and right target jumps found a highly significant effect of group $\left(\mathrm{F}_{2,19}=7.27, p<0.005\right)$, but no effect of side $(p=0.53)$, and no interaction $(p=$ $0.54)$. The neglect group successfully stopped their reaches on only $48 \%$ (SD 29) of jump trials, compared with $80 \%$ (SD 14) and $88 \%$ (SD 19) for RBDC and $\mathrm{HC}$ groups respectively. Although distinguishing the RBDN group overall, this impairment was unrelated to neglect severity within the group (correlation with BIT score: $r^{2}=0.08$ ). We propose that this finding reflects a non-lateralised deficit of response inhibition, which is not core to visual neglect, but which often accompanies the syndrome and may colour its expression [1].

\section{Acknowledgements}

SR supported by Portuguese Foundation for Science and Technology grants (numbers SFRH/BD/23230/ 2005, SFRH/BPD/65951/2009).

\section{References}

[1] S.H. Butler, S.R. Rossit, I.D. Gilchrist, C.J. Ludwig, B. Olk, R. Muir, I. Reeves and M. Harvey, Non-lateralised deficits in anti-saccade performance in patients with hemispatial neglect, Neuropsychologia 47 (2009), 2488-2495.

[2] M. Harvey, S.R. Jackson, R. Newport, T. Krämer, D. Llewlyn Morris and L. Dow, Is grasping impaired in hemispatial neglect? Behavioural Neurology 13 (2001), 17-28.

[3] M. Himmelbach and H.-O. Karnath, Goal-directed hand movements are not affected by the biased space representation in spatial neglect, Journal of Cognitive Neuroscience 15 (2003), 972-980.

[4] J.B. Mattingley, M. Husain, C. Rorden, C. Kennard and J. Driver, Motor role of human inferior parietal lobe revealed in unilateral neglect patients, Nature 392 (1998), 179-182.

[5] R.D. McIntosh, C.L. Pritchard, H.C. Dijkerman, A.D. Milner and R.C. Roberts, Prehension and perception of size in left visual neglect, Behavioural Neurology 13 (2001), 3-15.

[6] R.D. McIntosh, K.I. McClements, H.C. Dijkerman, D. Birchall and A.D. Milner, Preserved obstacle avoidance during reaching in patients with left visual neglect, Neuropsychologia 42 (2004a), 1107-1117.

[7] R.D. McIntosh, K.I. McClements, I. Schindler, T.P. Cassidy, D. Birchall and A.D. Milner, Avoidance of obstacles in the absence of visual awareness, Proceedings of the Royal Society of London B 271 (2004b), 15-20.

[8] A.D. Milner and R.D. McIntosh, Reaching between obstacles in spatial neglect and visual extinction, Progress in Brain Research 144 (2003), 213-226.

[9] L. Pisella, H. Grea, C. Tilikete, A. Vighetto, M. Desmurget, G. Rode et al., An 'automatic pilot' for the hand in human posterior parietal cortex: toward reinterpreting optic ataxia, Nature Neuroscience 3 (2000), 729-736.

[10] S. Rossit, P. Malhotra, K. Muir, I. Reeves, G. Duncan, K. Livingstone, H. Jackson, C. Hogg, P. Castle, G. Learmonth and M. Harvey, No neglect-specific deficits in reaching tasks, Cerebral Cortex 19 (2009), 2616-2624.

[11] T. Schenk, I. Schindler, R.D. McIntosh and A.D. Milner, The use of visual feedback is independent of awareness: evidence from visual extinction, Experimental Brain Research 167 (2005), 95-102. 


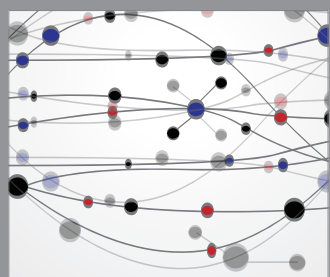

The Scientific World Journal
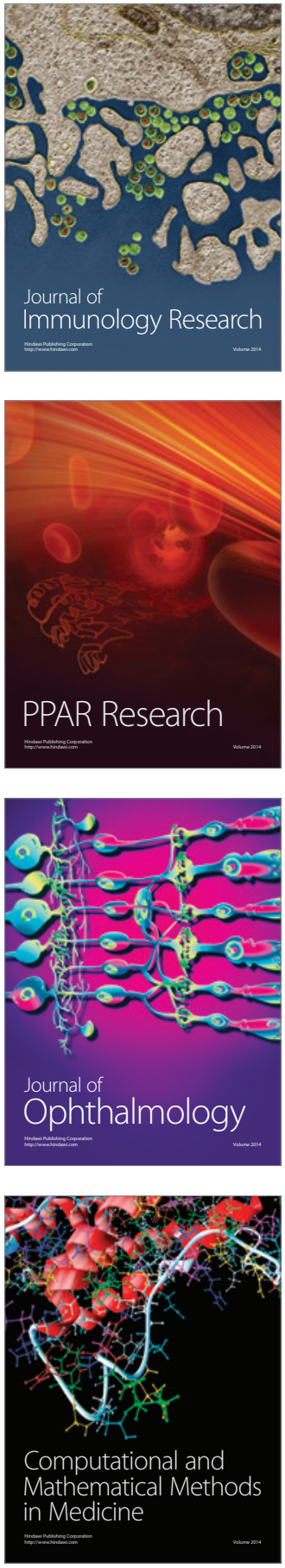

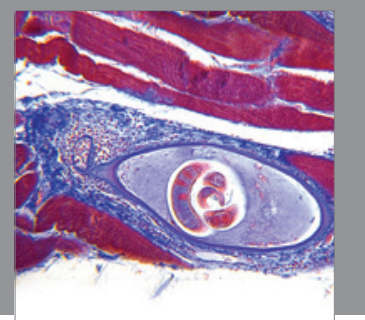

Gastroenterology

Research and Practice
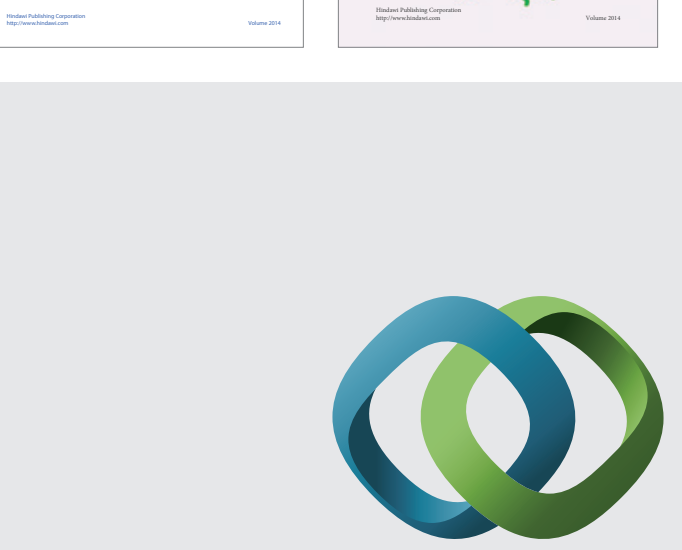

\section{Hindawi}

Submit your manuscripts at

http://www.hindawi.com
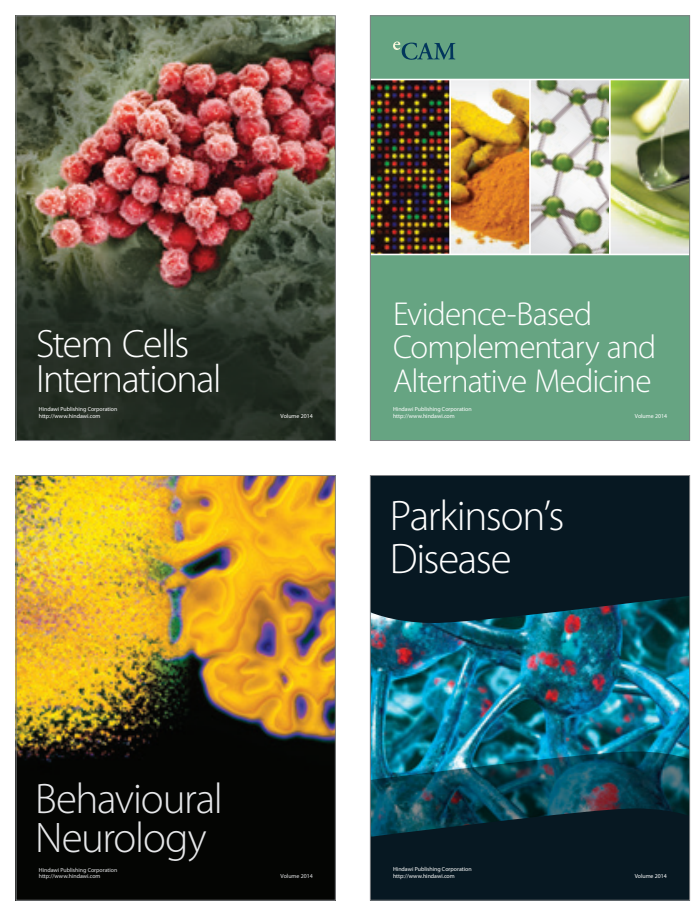

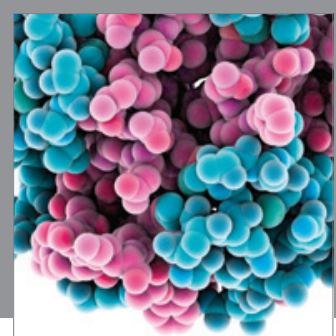

Journal of
Diabetes Research

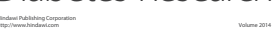

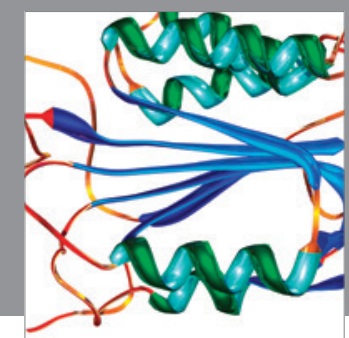

Disease Markers
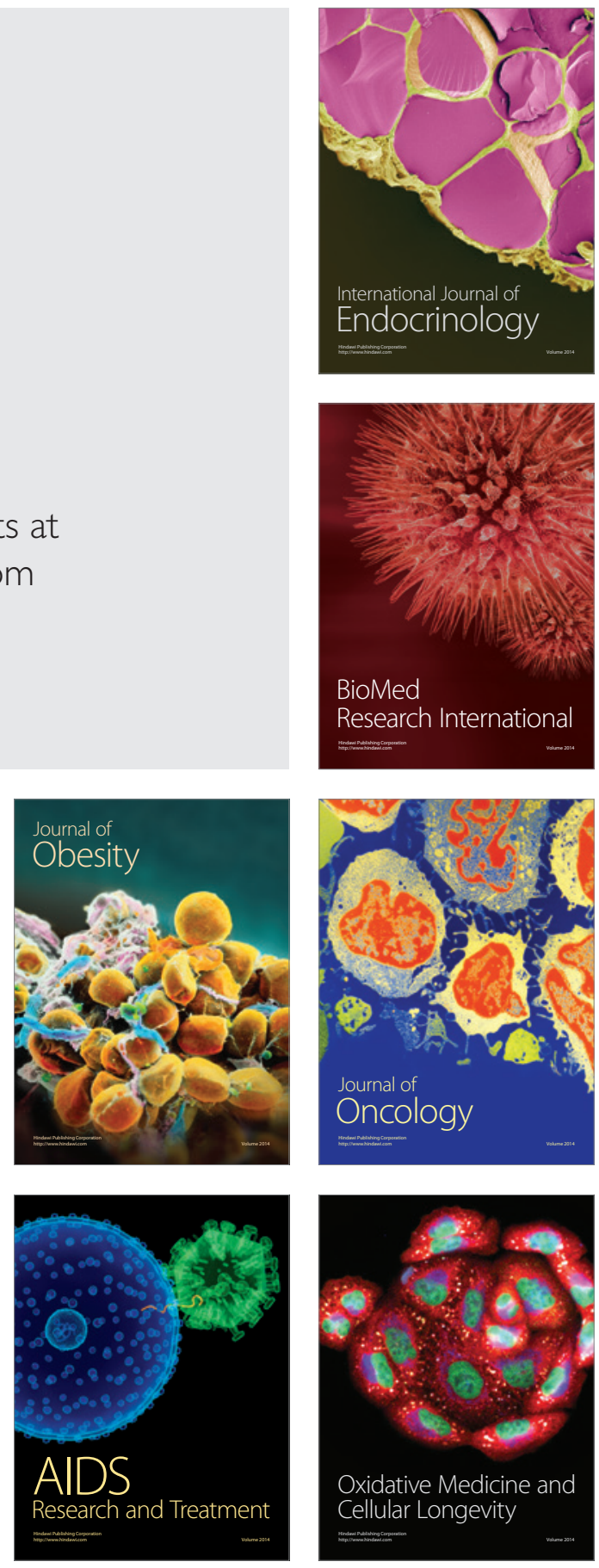